\title{
Forensische PopMUSIK-ANALYSE
}

\author{
Helmut Rösing
}

\section{Allgemeine Überlegungen zur Analyse von (Pop-)Musik}

Musikanalyse ist immer Interessen geleitet. Fragen zum Stil, zur musikgeschichtlichen Einordnung, zur Funktion und zur Zielgruppe, zur Qualität und zur Bewertung nach Kriterien wie Originalität, Authentizität, Emotionalität und Botschaft können Ausgangspunkt und Ziel einer analytischen Auseinandersetzung mit Musik sein. Im Mittelpunkt aber steht die Klärung der Machart von Musik. Zu deren Beschreibung gibt es unterschiedliche Verfahrensweisen.

Rein physikalisch lassen sich die Spektren von Schallereignissen nach Gegebenheiten wie Tonhöhenverlauf, Teiltonstruktur, Geräuschanteil und Zeitdauer der Schallereignisse, nach Intensitätsmerkmalen, Amplitudenschwankungen und Envelope-Struktur statistisch auswerten und benennen. Informationstheoretische und semiotische Modellbildung kann darauf aufbauen (Stoffer 1996).

Für eine musikalische Beschreibung sind derartige physikalische und psycho-akustische Parameter jedoch solange wenig ergiebig, wie sie nicht in Verbindung gebracht werden mit wahrnehmungspsychologischen Grundlagen des Musikhörens. Hier geht es um die Repräsentation von akustischen Reizen in den auditiven Verarbeitungszentren des Gehirns nach den Kriterien von dem, was als Musik gilt.

Damit erst beginnt die eigentliche Musikanalyse. Grundlage dafür bietet weniger deren physikalische Erscheinungsform als vielmehr ihre notenschriftliche Kodierung. Notenschrift hat sich in der westlichen Kultur seit dem Mittelalter von den Gestaltungsverläufe skizzierenden Neumen bis hin zur analytisch-sezierenden Einzelton-Notation entwickelt. Analog dazu wurde ein musiktheoretischer Überbau geschaffen, der in einem umfangreichen Regelwerk seinen Niederschlag gefunden hat. Diese Regeln sind im 
Verlauf der Musikgeschichte immer differenzierter und komplexer geworden. Das führte zu einer zunehmenden Kluft zwischen einer musiktheoretisch fundierten, nach Noten komponierten Kunstmusik und einer nach wie vor primär mündlich tradierten Umgangs-, Gebrauchs- und Volksmusik.

Popmusik ist allerdings weder Kunst- noch Volksmusik. In seinem Buch Die vier Weltalter der Musik beschreibt sie Walter Wiora als die im »Weltalter der Technik und globalen Industriekultur « aufgekommene »Musik für Hörermassen« (Wiora 1961: 125). Sie hat sich aus Gassenhauer, Volkslied, Operette und Schlager entwickelt und ist durch die Fusion mit afroamerikanisch ausgerichteten Musikstilen wie Blues und Rock, ferner durch die konsequente Einbeziehung aller jeweils zur Verfügung stehenden elektronisch-digitalen Gestaltungsmöglichkeiten zur dominierenden Kraft im aktuellen Musikgeschehen geworden.

Auch Popmusik basiert selbstverständlich auf den Grundlagen der abendländischen Musiktheorie, nicht aber auf dem für Kunstmusik typischen, hoch elaborierten theoretischen Überbau. Mit anderen Worten: Sie ist meistens tonal oder modal ausgerichtet, sie arbeitet mit Akkorden und Akkordfortschreitungen der klassischen Harmonielehre, sie verwendet einfache formale und satztechnische Strukturen, sie bevorzugt einen durchgehenden und eingängigen Beat, und sie huldigt dem Refrainprinzip mit Ohrwurmqualität: Musikalische Motive verdichten sich zu einer prägnanten Gestalt, deren Wiedererkennungswert mit jeder Wiederholung zunimmt. Jeder, der will, kann sich diesen Typ von Musik hörend aneignen - ohne musikalische Vorkenntnisse oder gar Expertenwissen.

Wie Rock, Jazz oder elektronische Tanzmusik zählt auch Popmusik zu jenen Musiksparten, die im herkömmlichen wissenschaftlichen Diskurs allein schon darum stigmatisiert sind, weil sie ohne notenschriftliches Substrat auskommen. Ihre Entstehung und Tradierung erfolgt oral bzw. medial. Die hier von Experten nach wie vor gebetsmühlenartig konstatierte >Regression der musikalischen Mittel < verkennt die Tatsache, dass es zumindest vier Aspekte sind, die es bei der Einschätzung von Popmusik gleichrangig zu berücksichtigen gilt: 1 . die Musik und ihre Faktur, 2. ihre Kommunikationsstrukturen, d.h. die Symbolhaltigkeit der Sounds und deren Bezug zu verschiedenen Lebenswelten, 3. die Marktsituation mit den konstanten Größen Geld und Macht sowie 4. die Präferenzen der Musikhörenden in Anlehnung an deren Wertbindungen und Lebensstile (Rösing 2002: 24f.).

Alle vier Aspekte ergeben eine Matrix wechselseitiger Bezugnahmen. Durch sie wird die Produktion von Musik ebenso bestimmt wie deren Distribution und Rezeption. Was als musikstrukturelle Regression bzw. Verfall abgetan wird, erhält in dem Bezugsfeld dieser Matrix einen ganz anderen 
Stellenwert als in der Kunstmusik. Je eindeutiger Popmusik zudem am Markt orientiert und kommerziell ausgerichtet ist, desto so mehr unterliegt sie dem Gesetz der Einfachheit. Strukturelle Simplizität statt Komplexität ist angesagt, um eine vertraute Symbolwelt musikalischer Klänge entstehen zu lassen. Für den Kunstmusik-Liebhaber ist das trivial im negativen Sinn. Für den Normalhörer aber hat diese Trivialität ihren besonderen Reiz. Durch sie werden die musikalischen Codes und Symbolwelten emotional verständlich, assoziativ nachvollziehbar und körperlich umsetzbar. Trivialität avanciert somit zu einem entscheidenden Qualitätsmerkmal.

Folgt man Peter Wicke, dann wird für den musikstrukturellen Bereich die »Materialität von Klang « zum Ausgangspunkt einer musikkulturellen Praxis, die auf »Sinnlichkeit, Vergnügen und Unterhaltung« zielt (Wicke 2002: 70). Somit geht es primär darum, die jeweiligen Charakteristika von Klang - verstanden als die Gesamtheit aller die sinnliche Qualität von Musik bestimmenden Faktoren - analytisch aufzuschließen.

\section{Forensische Popmusik-Analyse}

Der hier skizzierte Sachverhalt ist grundlegend für so gut wie jede Analyse von Popmusik, die von einem Gericht in Auftrag gegeben wird. Im Mittelpunkt steht dabei aber weniger eine umfassende stilistische, historische und gesellschaftliche Bewertung von Popmusik-Titeln in ihrer Gesamtheit als vielmehr die Frage nach dem Anteil der eigenschöpferischen Leistung an dem Musikprodukt.

Forensische Popmusik-Analyse ist immer vergleichend. Ausgangspunkt sind zwei Musikstücke: ein urheberrechtlich geschütztes Original und ein später entstandenes Stück, in dem angeblich urheberrechtlich geschütztes Material Verwendung gefunden hat. Was der Kläger diesbezüglich vorbringt, versucht die Seite der Beklagten zu widerlegen.

Die Argumentation des Klägers vollzieht sich in der Regel in zwei Schritten, um eine Urheberrechtsverletzung so plausibel wie möglich nachzuweisen. Erstens muss deutlich gemacht werden, dass das Originalwerk eine eigenschöpferische Qualität und Wertigkeit besitzt. Grundlage dafür ist die minutiöse Analyse kleinster Musikpartikel, die aus kunstmusikalischer Sicht als eher trivial oder banal abgetan zu werden pflegen, hier aber als Indikatoren für musikalische Prägnanz stehen: Aus musikstrukturellen >Kleinigkeiten k kann eine Motivsemantik erwachsen, die für geistiges Eigentum kennzeichnend ist. Zweitens ist darzulegen, dass musikalische Bestandteile, die nachweislich zur schöpferischen Individualität des Originalstücks beitra- 
gen, auch in jenem Musiktitel zu finden sind, dem Urheberrechtsverletzung vorgeworfen wird.

Die Abwehrstrategie der Beklagtenseite besteht darin, diese Eigentümlichkeiten klein zu reden. Als Beleg wird gerne auf die Nähe zu volkstümlichem und damit urheberrechtsfreiem Liedgut verwiesen. Oder aber auf angeblich vergleichbare Partien aus so genannten klassischen Kompositionen. Im Vergleich mit deren Strukturen wird dann die Machart des Poptitels als banale Anwendung rein handwerklicher Musikbetätigung abgetan. Dass eine derartige Argumentationsweise dem Untersuchungsgegenstand Popmusik nicht gerade angemessen ist, dürfte nach den bisherigen Ausführungen zweifelsfrei sein.

Auslöser für eine gerichtliche Auseinandersetzung mit in Deutschland geschaffener Popmusik ist der Paragraph 24 des deutschen Urheberrechts vom 1. Januar 1966 (im Überblick Brösche 1998; ausführlich im Hinblick auf Popmusik Pendzich 2004: 147ff.). Ausschlaggebend für eine gerichtliche Auseinandersetzung sind letztlich so gut wie immer finanzielle Aspekte: Wer ist der legitime Nutznießer der Umwandlung von geistigem Eigentum in klingende Münze? Wem gehört die fragliche Musik, ein zündender musikalischer Einfall, ein bestimmter Sound, ein markanter Basslauf, ein rhythmisch auffallend gebautes Motiv oder eine Melodie mit hohem Wiedererkennungswert? Handelt es sich hier um die kompositorische Verwertung von musikalischem Allgemeingut, um den gekonnt handwerklichen Umgang mit Ton-, Rhythmus- und Akkordfiguren zur Schaffung eines individuell geformten neuen Musikstücks, oder handelt es sich um unfreie Anleihen bei bereits vorhandenen und im Rahmen der gesetzlichen Fristen urheberrechtlich geschützten Musikstücken? Pointiert gesagt: Ein Plagiatvorwurf wird erhoben, wenn es sich lohnen könnte, am kommerziellen Erfolg eines Musikstücks zu partizipieren.

Die juristische Klärung derartiger, um einen Plagiatsvorwurf kreisenden Fragen ist zwar durch das Urheberrecht geregelt, bedarf aber - soweit es die Analyse und Einschätzung der musikimmanenten Sachverhalte betrifft der außergerichtlichen Expertise. Doch auch sie ist letztlich den Festschreibungen im Paragraphen 24 verpflichtet. Der hier in Absatz 2 explizit formulierte Melodienschutz macht deutlich, dass dieses Gesetz seine geschichtlichen Wurzeln im 19. Jahrhundert hat. Die Melodie - definiert als in sich geschlossene Tonfolge, die dem Musikwerk seine individuelle Prägung gibt galt zu dieser Zeit noch als das höchste Gut der westlichen Musik und somit als der entscheidende Indikator für schöpferische Leistung. Ein starrer Melodienschutz aber wird den musikimmanenten Gegebenheiten von Popmusik nur bedingt gerecht. 
Nicht zuletzt auch aus diesem Grund wurde das Melodiendiktat schon 1921 von Alexander Elster durch den Begriff der »Kleinen Münze« wenn schon nicht aufgehoben, so doch zumindest relativiert (Elster 1921). Die Kleine Münze bezeichnet die unterste Grenze eines gerade noch urheberrechtlich geschützten und zu schützenden Werkes. Es benennt allerdings keine klaren Kriterien hinsichtlich der Minimalanforderungen an die allgemeine und die melodische Gestaltungshöhe. Der BGH hat dazu jedoch in den 1980er/1990er Jahren einige Grundsatzurteile mit Präzedenzwirkung gefällt. ${ }^{1}$ Bereits kleinste melodische und rhythmische Veränderungen von musikalischem Allgemeingut stellen demnach einen bewussten kompositorischen Eingriff dar und sind die Grundlage für die Schaffung von geistigem Eigentum. Kritiker meinen, damit sei die kompositorische Freiheit eingeschränkt, weil es praktisch keine allgemeinfreien Musikbausteine mehr gebe. Die Befürworter verweisen jedoch darauf, dass gerade für Popmusik die Kleine Münze ein höchst angemessenes Beurteilungskriterium darstelle. Dies vor allem dann, wenn der musikalische Gesamteindruck ${ }^{2}$ zum Maß für eigenschöpferische Leistung erhoben wird.

Der Gesamteindruck ist das Ergebnis eines Ineinandergreifens von mindestens fünf Gestaltungsebenen:

- Strukturebene (großformale Anlage, Periodenbildung, Wiederholung, Taktaufteilung);

- klangliche Ebene (Klangfarbe als Ergebnis von Instrumentation und Arrangement, elektronische Soundgestaltung);

- rhythmische Ebene (Tempo, zeitliche Dichte der Tonfolgen, Zeitdauer und Betonung der Töne im Taktgefüge);

- melodische Ebene (Anordnung von Tonfolgen - Tonhöhen, Intervalle zu einer in sich geschlossenen Gestalt);

- harmonische Ebene (Zusammenklänge - in tonaler Musik nach den Regeln der Harmonielehre).

Die Gewichtung der fünf Gestaltungsebenen ist je nach Gattung und Stil der Popmusik unterschiedlich anzusetzen. Bei Techno z.B. steht die klangliche, bei HipHop die rhythmische und beim volkstümlichen Schlager die melodi-

1 5.9.1970: »Magdalenenarie« (Az. I ZR 44/68 Kammergericht Berlin); 26.9.1980: »Dirlada« (Az. I ZR 17/78 OLG Hamburg); 3.2.1988 »Fantasy« (Az. I ZR 143/86 Kammergericht Berlin); 3.2.1988: »Ein bißchen Frieden« (Az. I ZR 142/86 Kammergericht Berlin); 24.1.1991: »Brown Girl I« (Az. I ZR 78/89 OLG Hamburg); 24.1.1991:»Brown Girl II« (Az. I ZR 72/89 OLG Hamburg).

2 Siehe die Urteilsbegründung zu »Brown Girl II« in Gewerblicher Rechtschutz und Urheberrecht (GRUR) 1991: $533 \mathrm{f}$. 
sche Ebene im Fokus der Komposition. Schon dieser Umstand lässt deutlich werden, dass die Kriterien zur Bestimmung von eigenschöpferischer Leistung gemäß der Kleinen Münze nicht nach festen Richtlinien dingfest gemacht werden können. Sie müssen von Einzelfall zu Einzelfall neu verhandelt und der jeweiligen Musikform in angemessener Weise zugrunde gelegt werden. Das soll im Folgenden anhand von vier Beispielen näher erläutert werden.

\section{Einzelanalysen}

\section{»Ich bin hier, du bist da « $\quad$ »FKK«}

Ende der 1980er Jahre haben Jürgen Dohrenkamp alias Jürgen von der Lippe und Wendelin Haverkamp das Lied »Ich bin hier, du bist da «komponiert und getextet. Es erschien 1990 mit Jürgen von der Lippe als Sänger auf der CD Humor ist Humor. Vier Jahre später wurde von BMG eine CD mit dem Titel Lieder, die die Welt nicht braucht veröffentlicht. Sie enthält u.a. das Lied »FK« «, komponiert und gesungen von Die Doofen (Wigand Boning und Oliver Dittrich). Hier nun war zu klären, ob erstens der Refrain des Liedes »Ich bin hier, du bist da « wirklich eigenschöpferische Elemente aufweist und zweitens die Übereinstimmungen der Refrains in den Liedern »Ich bin hier, du bist da« und »FKK« so groß sind, dass eine Urheberrechtsverletzung vorliegt.

(1) Das Lied »Ich bin hier, du bist da « beginnt nach kurzer Instrumentaleinleitung mit dem Refrain. Er steht im 4/4-Takt, umfasst acht Takte und ist nach jeder der vier Liedstrophen unverändert zu hören (Notenbeispiel 1). Am Ende erklingt er ein weiteres Mal in einer schlusstypischen Variante. Das ist formal gesehen Standard. Auch harmonisch greift der Refrain auf Bekanntes zurück. Er steht in D-Dur, weist keine harmoniefremden Töne auf und folgt dem Ablauf T-D-D-T (Takt 1-4) bzW. T-S-D-T (Takt 5-8):

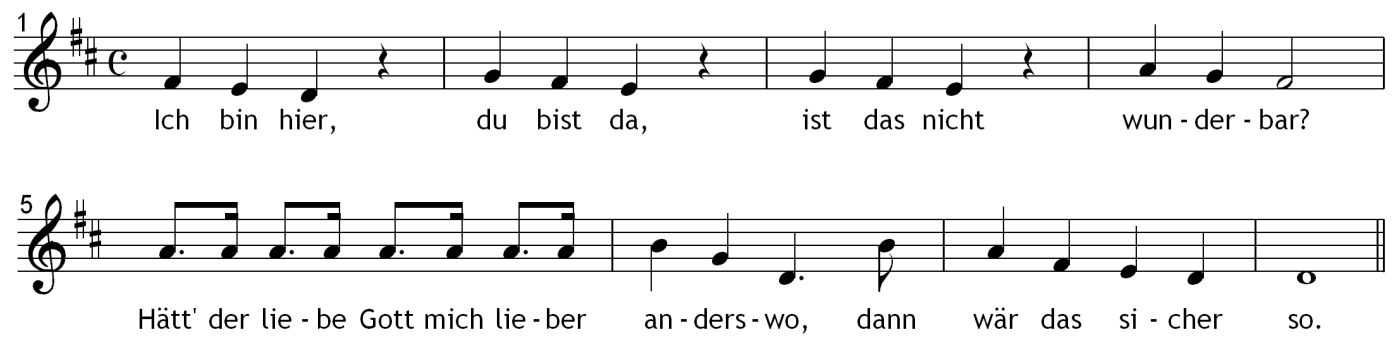

Notenbeispiel 1: Jürgen von der Lippe und Wendelin Haverkamp - »lch bin hier, du bist da«, Refrain 
Das entscheidende, vom Sänger vorgetragene motivische Material des gesamten Refrains ist ein dreitöniges, abwärts geführtes Terzmotiv in eingestrichener Tonlage. Es wird in den Takten 1-4 von fis, $g$ und $a$ ausgehend gebracht. Da es sich um eine tonale Sequenzierung handelt, ändert sich die Intervallstruktur entsprechend dem Ausgangston. Die Variantenbildungen in der Intervallstruktur des Kernmotivs sind kein Ergebnis einer kompositorischen Leistung. Sie ergeben sich vielmehr zwangsläufig. Gleichwohl stellen sie ein belebendes Element dar. Das zwischen aufsteigender Quarte und kleiner Terz changierende Übergangsintervall zwischen den Terzzügen dagegen ist sehr wohl das Resultat einer kompositorischen Entscheidung.

Takt 5 bringt im Parlando-Stil auf dem Ton $a$ den Anlauf zum Höhepunkt der Refrainmelodie, dem $h$ in Takt 6. Damit umspannt die Refrainmelodie den Umfang einer Sexte und verweist auf die Nähe zu volkstümlichen Liedgut. Der Sext-Ambitus wird zum Schluss von Takt 6 durch den Sextsprung aufwärts als einzigem emphatischem Element in der ansonsten betont ruhig gehaltenen Melodieführung besonders hervorgehoben, bevor in Takt 7 der Abstieg zum Grundton erfolgt. Dabei kommt nochmals das Kernmotiv des Refrains zu Gehör. Nicht zuletzt dank dieses motivischen Rückbezugs enthält der Refrain jene Geschlossenheit, die die Motivfolge zu einer Melodie werden lässt.

Das rhythmische Geschehen und die klangliche Gestaltung sind konsequent einfach gehalten. Im stimmlichen Vortrag wird trotz gemäßigtem Tempo auf Modulationsreichtum oder gar Dramatik bewusst verzichtet. Der Gesangsvortrag wirkt insgesamt zurückgenommen. Die Einwürfe des Background-Chores erinnern ebenso wie das gesamte Arrangement mit kurzen Floskeln von Hawai-Gitarre und Mundharmonika an Versatzstücke aus Schlagern der 1960er Jahre, aber eben nur an Versatzstücke ohne den dort üblichen stimulierenden Impetus.

Das alles passt zusammen. Die verschiedenen musikstrukturellen Ebenen zeichnen sich durch große Einfachheit, durch bewusste Reduktion und durch die Vermeidung von dramatischen Akzenten aus. Das der Volksmusik entlehnte Hauptmotiv hat Ohrwurmqualität und animiert zum Mitsingen. Dem wird aber durch die Monotonie des Refrainverlaufs ein Dämpfer versetzt. Aus dieser Spannung zwischen Erwartetem und tatsächlich Realisiertem bezieht der Refrain seine ihm eigene ästhetische Qualität.

(2) Das Lied »FKK« hat demgegenüber einen ganz anderen Charakter. Es weist ein zügiges Tempo auf und beginnt mit Schlagzeugbreak und instrumental gespieltem Refrain mit dissonierender Flöte zu Schlagzeug, Bass und Akkordeon. Eingeleitet durch ein stimulierendes »Hey« des Vokalduos wird 
sodann die achttaktige Refrainmelodie gesungen. Sie ist ebenso wie ihre harmonische Ausgestaltung tonal gehalten:

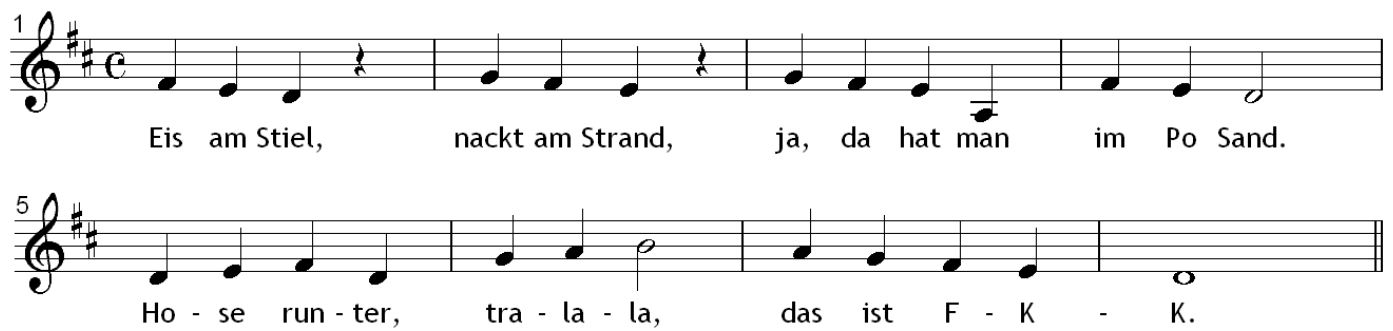

Notenbeispiel 2: Die Doofen - »FKK«, Refrain

Das von den zwei Sängern vorgetragene motivische Material der ersten Hälfte des Refrains besteht aus dem vom vorigen Lied bestens bekannten Kernmotiv. Es erklingt viermal - ebenfalls in eingestrichener Lage. Zwischen dem 3. und 4. Terzmotiv wird jedoch das tiefe $a$ angesungen. Das Aufwärtsintervall zum Anfangston fis des 4. Terzmotivs beträgt eine Sexte. Das führt zu einer deutlichen binnenstrukturellen Dynamisierung dieses 1. Refrainteils, der folgerichtig auf dem Grundton $d$ als Schlusston endet. Im 2. Refrainteil ertönt das Terzmotiv zweimal in der Umkehrung, wird also aufwärts statt abwärts geführt.

Somit weist der Refrain von »FKK« erhebliche Unterschiede im Vergleich mit dem Refrain von »Ich bin hier, du bist da « auf. Sie werden durch die weiteren Strukturebenen noch verstärkt. Der Rhythmus verfügt über eine dynamisierende, fast schon gehetzt-treibende Kraft. Er ist eher dazu angetan, die durchweg skuril-komischen Textaussagen im Sinn einer schnellen Pointenfolge zu unterstreichen. Dabei wird auf stiltypische Versatzstücke aus Swingzeit und Dixieland-Revival zurückgegriffen.

Bleibt als Fazit: Die vorrangig, aber keineswegs ausschließlich melodiebezogene Detailanalyse der musikalischen Struktur beider Refrains zeigt neben deutlichen Übereinstimmungen auch erhebliche Unterschiede auf. Sie betreffen alle Gestaltungsebenen. Selbst die teilweise vorhandene Identität der verwendeten Motive führt keineswegs zur Identität der Refrainmelodien. Beiden Stücken kann eine individuelle Prägung im Sinn der Kleinen Münze zugesprochen werden. Der Vorwurf des Plagiats erweist sich als ungerechtfertigt. 


\section{»Mambo No. 5« - »Mambo No. 5 - A Little Bit Of...«}

Im Jahr 1999 veröffentlichte BMG den Titel »Mambo No. 5 - A Little Bit Of...« von Lou Bega. Die CD wurde ein Verkaufsschlager. Nicht zuletzt aus diesem Grund erhob sich die Frage, ob es sich hier um eine selbstständige Komposition handelt oder um eine Adaption eines nahezu gleich lautenden Stücks aus den 1950er Jahren: »Mambo No. 5 « des kubanischen Komponisten, Arrangeurs und Bandleaders Perez Prado.

Von Prados »Mambo No. 5« liegen acht Tonträger vor, auf drei von ihnen sind unterschiedliche Versionen des Stücks eingespielt. Eine konstante Größe hierbei stellen allerdings drei Bläserriffs dar. Sie geben dem Stück seine eigentliche Kontur. Die folgende Analyse basiert auf der Version, wie sie der Sampler Perez >Prez Prado: King of Mambo enthält, da sie fraglos als Vorlage für den Mambo-Titel von Lou Bega hergehalten hat.

Riff 1 eröffnet das Stück (siehe Notenbeispiel 3 auf der folgenden Seite). Es besteht aus vier aufsteigenden, jeweils halbtaktigen Dreiklangsbrechungen. Die Aufwärtsbewegung wird in der zweiten Takthälfte durch einen Sekundschritt abwärts gestoppt und mit dem folgenden Sekundschritt aufwärts auf dem letzten Achtel zur Eins des nächsten Taktes übergeleitet. Die rhythmische Struktur ist geprägt durch Akzente auf dem 1. Viertel sowie dem 6. Achtel; der synkopische Charakter wird zudem durch die Pause auf dem 3. Achtel verstärkt. Dieses Riff kann man für afro-kubanische Musik als durchaus gängig und damit allgemeinfrei bezeichnen.

Anders verhält es sich mit Riff 2 . Es besteht aus einem mehrstimmigen Bläsersatz, in dem zwei in ihrem Verlauf gegensätzliche Motive miteinander verzahnt sind. Motiv A wird gespielt von zwei Trompeten und Altsaxophon, Motiv B von Tenor- und Baritonsaxophon. Motiv A sspielt < mit der kleinen Terz, die in jeweils einem Takt aufwärts und dann wieder abwärts geführt wird. Motiv B ist gekennzeichnet durch einen Quartsprung aufwärts, Quintsprung abwärts / aufwärts sowie einen Quartsprung abwärts / aufwärts. Diese agile Bewegungsfigur erhält durch die rhythmische Schwerpunktverlagerung gegenüber Motiv A eine besondere Prägnanz. Die Akzente der Motive A und B sind mehrmals um ein Achtel versetzt. Das verleiht Riff 2 eine außerordentliche Dynamik, die durch Riff 3 mit zwei Viertelnoten auf dem 1. und 4. Viertel des Takts sehr effektvoll gestoppt wird. Für sich allein betrachtet, ist dieses Riff natürlich nicht schutzwürdig, sehr wohl aber im gesamten Kontext des Stücks durch seine Kombination mit Riff 2.

»Mambo No. 5 - A Little Bit Of...« ist eine Reminiszenz an die »gute, alte«Mambozeit und huldigt einem seiner herausragenden Repräsentanten, 

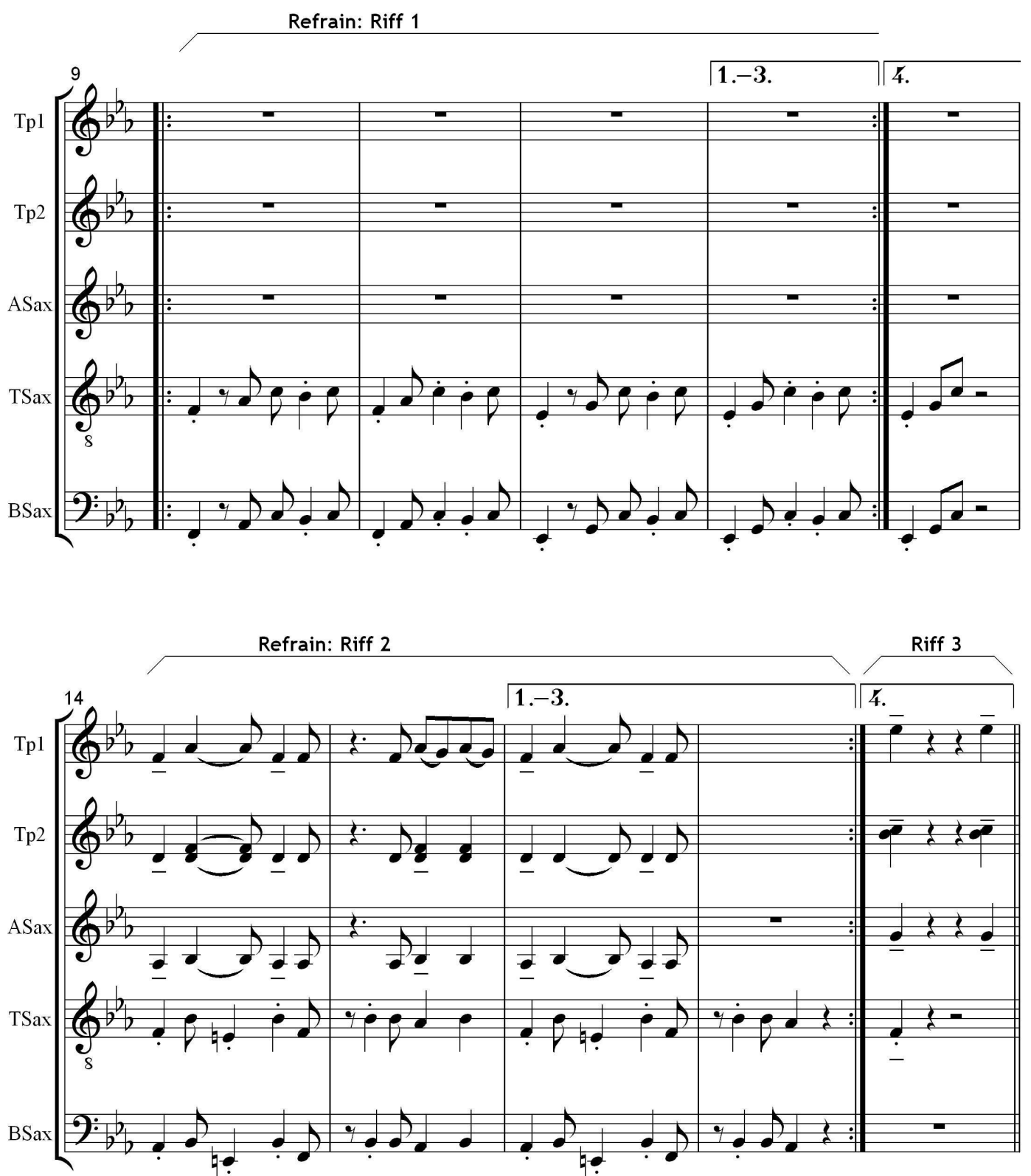

Notenbeispiel 3: Perez Prado - »Mambo No. 5«, Riff Nr. 1, 2 und 3

Perez Prado als »King of Mambo«. Der Titel von Prado gibt den Rahmen für das Stück von Lou Bega. Tempo, Mamborhythmik, Tonart und Harmonik (zwischen B und Es pendelnd) entsprechen der Vorlage, deren Riffs dem die Strophen abschließenden Gesangsrefrain jeweils in originaler Gestalt als instrumentale Begleitfigur unterlegt bzw. hinzugefügt wurden.

Riff 2 und 3 erklingen nach Introduktion, Strophe und Refraingesang in voller Lautstärke. Die während des Gesangs kontinuierlich gesteigerte Spannung entlädt sich im Schlussteil auf markante Weise gemäß den Worten des Songtextes »Please set in the trumpet«. Mit den gesampleten Prado-Riffs ist zugleich auch ein Bezug zur instrumentalen Introduktion des Mambo-Songs 
gegeben. Der Beginn des Stücks markiert mit Technosounds die musikalische Gegenwart, der Schluss dagegen den typischen Mambo-Sound der 1950er Jahre.

»Mambo No. 5 - A Little Bit Of... « von Lou Bega ist - daran lassen Introduktion, Strophen- und Refraingesang keinen Zweifel - ein eigenschöpferisches Werk. Seine Prägnanz aber verdankt es, so paradox das klingen mag, gerade dem Umstand, dass in ihm bestimmte kompositorische Elemente des Tanzstücks »Mambo No. 5« von Perez Prado nicht verblassen, sondern im Gegenteil ganz besonders hervorgehoben werden, und zwar im instrumentalen Schlussteil des Refrains durch die Eins-zu-Eins-Übernahme von Riff 2 und 3.

Urheberrechtlich lässt sich dieser Sachverhalt folgendermaßen präzisieren: Auf 16 Takte Original-Lou-Bega-Komposition kommen acht Takte Original-Perez-Prado-Komposition. Rein rechnerisch entspricht das einer Kompositionsleistung, die zu etwa 67 Prozent Lou Bega und zu 33 Prozent Perez Prado zuzuordnen ist.

\section{$\gg$ Let It Rain «}

Im Jahr 1997 wurde wiederholt in Funk und Fernsehen ein Werbespot für Uniroyal-Regenreifen gesendet, ${ }^{3}$ von dem es hieß, er enthalte musikalisches Material aus dem Song »Let It Rain« der US-amerikanischen Komponistin, Arrangeurin und Sängerin Kristen Hall. Die ohne Einwilligung der RechteInhaberin vorgenommene Bearbeitung für den Spot schien allerdings nicht auf der 1994 auf der CD Be Careful What You Wish For veröffentlichten Originalversion zu fußen, sondern auf der Refrainvariante einer 1995 für Sony von Amanda Marshall eingesungenen Coverversion. Es galt nun zu klären, ob der Refrain überhaupt schutzfähig ist und ob - wenn ja - seine Eigentümlichkeit im Spot erhalten geblieben ist.

Der Song »Let It Rain« steht dem afro-amerikanischen Blues- und SoulIdiom nahe. Das erfordert, in Bezug auf den Gesang, eine stimmlich markante und vor allem im Refrain möglichst emphatische Gesangsweise. Ohnehin variieren von Strophe zu Strophe und Refrain zu Refrain Stimmgebung, Tonfall und melodische Verzierungen im Sinn von Paraphrase bzw. Improvisation auf der Grundlage eines durch die melodische Kontur, durch Tempo, Rhythmus und Takt sowie die harmonische Abfolge vorgegebenen Verlaufsschemas. Das alles lässt sich notenschriftlich wegen ihrer »drastischen Ab-

3 Produziert von Tobias Gad für Daydream Film Productions München (1997). 
straktion« und Klangneutralität nur rudimentär festhalten (Schneider 2002: 108).

Noch mehr als für das Original trifft dieser Sachverhalt auf die Coverversion zu. Das verdankt sich der ausdrucksstarken und modulationsfähigen Stimme von Amanda Marshall und betrifft nicht nur die Strophen, sondern natürlich auch den 16 Takte umfassenden Refrain (Notenbeispiel 4). Er wird insgesamt viermal gesungen und zwar jedes Mal ein wenig verändert. Seiner Einprägsamkeit und seinem Wiedererkennungswert tut das keinen Abbruch. Im Gegenteil, die bewusst einfach gehaltene motivisch-rhythmische Gestaltung des Refrains auf der Grundlage des gängigen Kadenzschemas Tonika Dominante - Subdominantparallele - Subdominante erhält gerade durch die improvisatorische Variantenbildung (Tonhöhenvarianten und -modulationen, ternäre Phrasierungen innerhalb des binären Taktschemas, stimmliche Farbschattierungen) den Reiz des Individuellen.
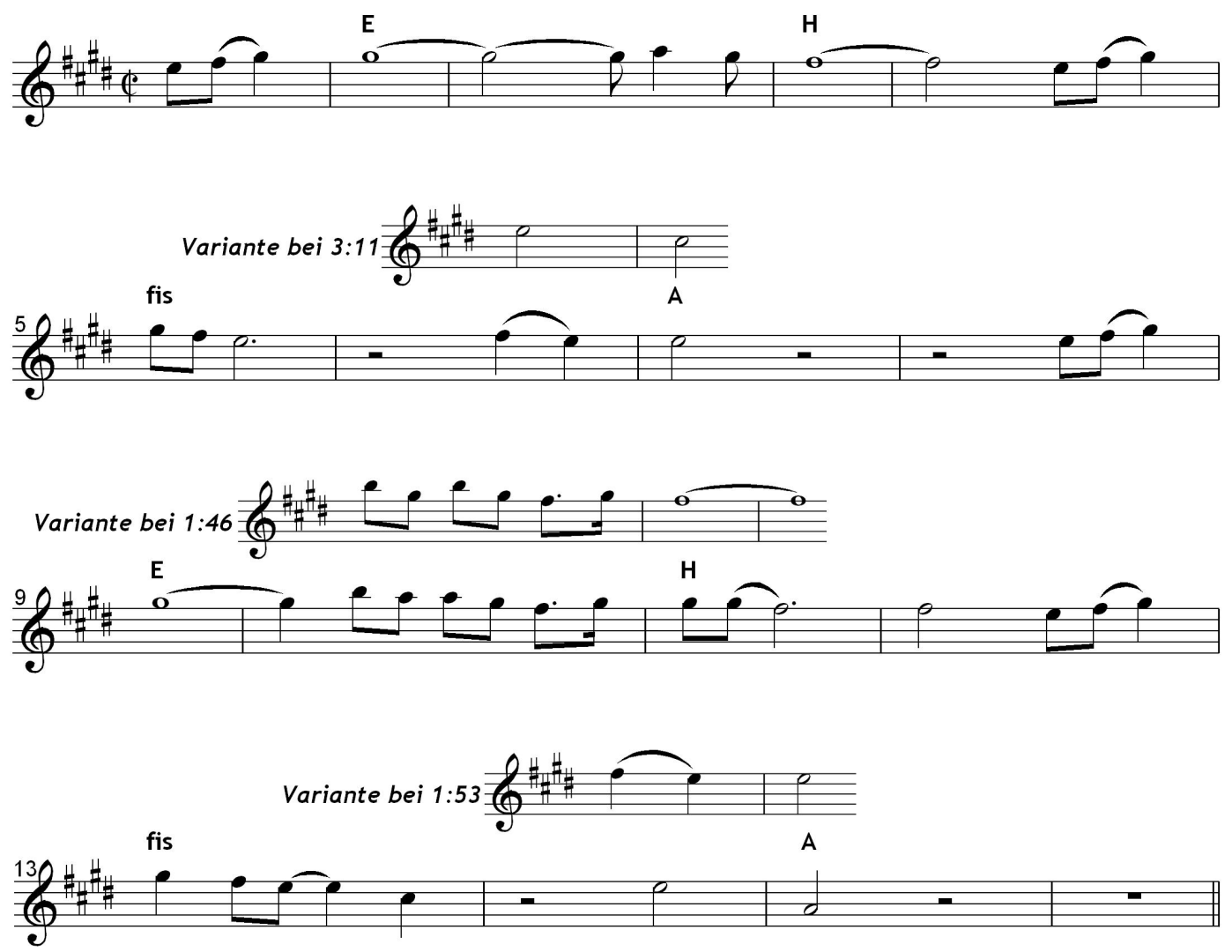

Notenbeispiel 4: Amanda Marshall — »Let It Rain«, Refrain

Die eigenständige kompositorische Leistung des Refrains im GitarrenpopArrangement allerdings liegt - gemessen an den Standards, die etwa hinsichtlich der melodischen Prägnanz eines Schlagers oder Evergreens anzusetzen sind - unterhalb des Niveaus der Kleinen Münze. Bezieht man aber 
die improvisatorische Züge tragende Interpretation in die kompositorische Leistung im weiteren Sinn mit ein, dann weist die von Amanda Marshall gesungene Coverversion durchaus individuelle, eigenschöpferische Gestaltungsmerkmale auf.

Für den Werbespot wurde die Refraingestaltung von Amanda Marshall als Vorbild und musikalischer Prototyp verwendet. Dabei war man bestrebt, eine Urheberrechtsverletzung zu umgehen. Das geschah vor allem durch die Veränderung der melodischen Kontur der Singstimme:
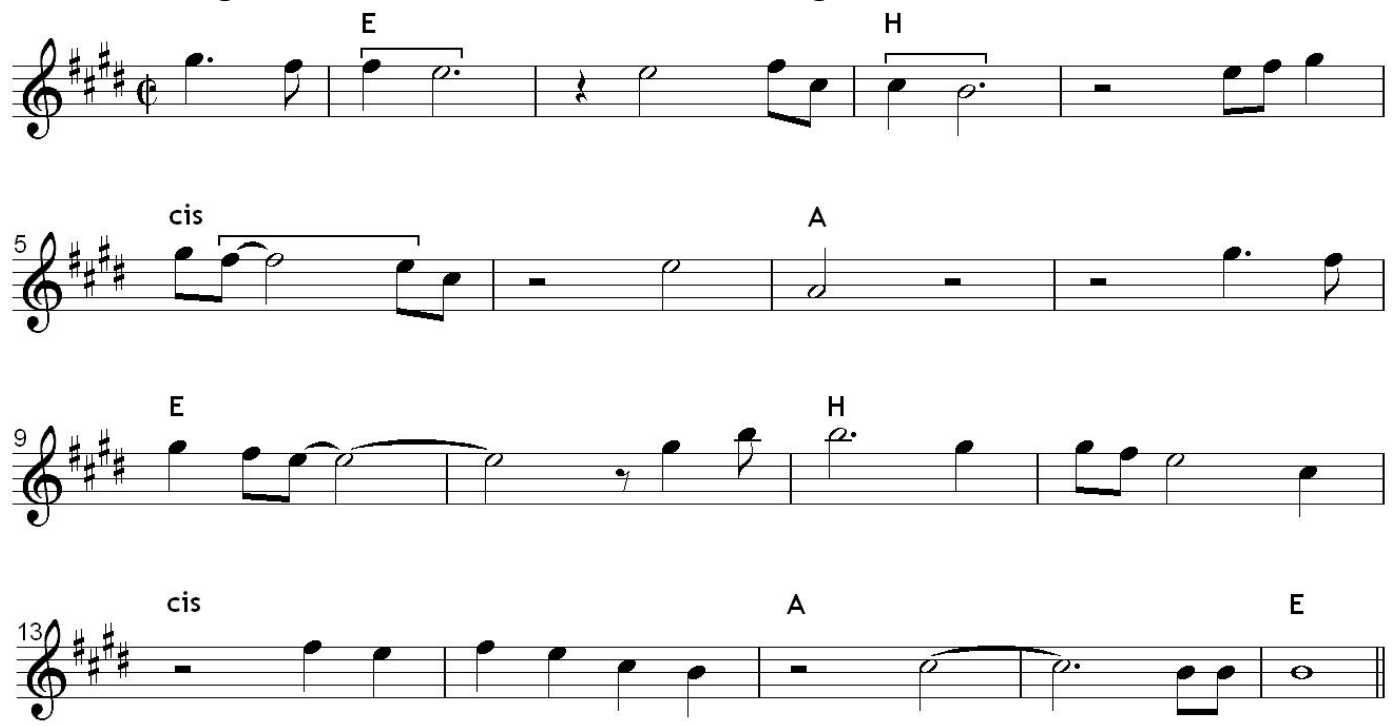

Notenbeispiel 5: Uniroyal-Werbespot

Über dem nahezu identischen Kadenzschema als harmonischem Grundgerüst wurden bei der Musik zum Uniroyal-Werbespot in der Tat mehrfach andere akkordeigene Töne zur Gestaltung des Verlaufs der Singstimme verwendet als im Refrain der Coverversion von Amanda Marshall. So ist z. B. der melodische Duktus der ersten Viertaktgruppe abwärts statt aufwärts gerichtet. Außerdem wird der Schlüsseltext »Let It Rain« zweimal statt nur einmal gesungen. Die zweite Viertaktgruppe dagegen kann als nahezu identisch bezeichnet werden. Sie weist geringfügige Veränderung bei den Tondauern auf und fußt auf der Tonikaparallele cis-Moll statt der Subdominantparallele fis-Moll.

Der Spannungsbogen beider Refrains erreicht seinen Höhepunkt übereinstimmend in der dritten Viertaktgruppe: bei Amanda Marshall auf dem zweigestrichenen $h$ im 2. Viertel von Takt 10, beim Spot ebenfalls auf dem $h$ im letzten Achtel von Takt 10. Der auf dem 4. Viertel von Takt 11 beginnende Abwärtszug gis-fis-e-cis in der Uniroyal-Musik stimmt mit der Intervallfolge in Takt 13 der Vorlage von Amanda Marshall überein. Das alles reicht aber nicht aus, um von einer direkten Melodieentnahme zu sprechen. 
Betrachtet man jedoch das Zusammenwirken aller am Musikgeschehen beteiligten Ebenen, dann ergibt sich ein ganz anderes Bild. Denn wirklich identisch sind die Tonart (E-Dur), die formale Anlage (Refrain mit vier Viertaktgruppen), der Takt (4/4), das gemäßigte Tempo, der Rhythmus (swingend), der Sound und das Arrangement (Gitarrenpop), der Schlüsseltext (»Let It Rain«) und die stimmliche Darbietung im soulnahen emphatischen Gesangsstil. Die Vorlage verblasst angesichts dieser Übereinstimmungen aller im Werbespot gezielt vorgenommenen geringfügigen Abänderungen zum Trotz - keineswegs beim vergleichenden Hören. Der ästhetische Gesamteindruck von Original und Kopie ist nahezu identisch. Damit bewahrheitet sich ein alter Grundsatz der Gestaltpsychologie: Die einzelnen musikalischen Elemente fügen sich zu einem Gesamteindruck mit einer bestimmten übersummativen Qualität zusammen. Diese kann auch dann unverändert bleiben, wenn einige Details nicht gleich sind.

Der Uniroyal-Werbespot wurde als besonders eklatante Ausbeutung von geistigem Eigentum bewertet. Er durfte nicht mehr gesendet werden und zudem wurde eine erhebliche Entschädigungssumme an die Inhaber der Urheberrechte fällig.

\section{»Winds Of The Lost Soul« — »Ghettorap hin, Ghettorap her «}

Im Jahr 2005 erschien bei Urban/Universal Music die CD Carlo Cokxxx Nutten 2 von Bushido. Sieben der insgesamt 15 Titel verwenden Samples der schwedischen Dark Wave-Band Arcana aus den Jahren 2000 bis $2004^{4}$, ohne dass dies auf der CD angegeben ist. Um die urheberrechtliche Relevanz dieser Übernahmen zu klären, waren die folgenden Fragen zu beantworten: 1. handelt es sich bei den Stücken von Arcana um urheberrechtlich geschützte Werke, 2. stellen die entnommenen Teile schutzfähiges Material dar, 3. handelt es sich hierbei um Kopien oder um bearbeitetes Material und 4. welche Funktion haben diese übernommenen Teile in den Stücken von Bushido?

(1) Die Musiktitel der Gruppe Arcana weisen eine klare großformale Anlage auf. Nach dem kompositorischen Baukastenprinzip erfolgt im Mehrspurverfahren eine zunehmende Schichtung recht verschiedener, in sich periodisch gegliederter Bausteine. Die Schichtung führt zu einer immer dichteren Textur im Stückverlauf. Die Lautstärke nimmt zu, der Klangraum weitet sich, und wiederholt führen glockenartige Klänge zu einem Maximum an Span-

4 Veröffentlicht auf The Last Embrace (2000), Inner Pale Sun (2002) und The New Light (2004). 
nungssteigerung, bevor die groß angelegten Spannungsbögen in einem schnellen Fade-Out zu ihrem Ende kommen.

Die einzelnen musikalischen Komponenten sind klanglich wie rhythmisch unterschiedlich und abwechslungsreich geformt. Zum Stückbeginn dominieren Bausteine mit kürzeren Tonbewegungen und Spielfiguren. Sie werden durch Motivkomponenten mit längeren Notenwerten und zunehmend melodischer Ausprägung überhöht. Die rhythmisch stärker bewegten Einleitungsfiguren bleiben im Hintergrund erhalten und verleihen dem nun eher großflächigen Gesamtklang eine unüberhörbare Lebendigkeit.

Diese Lebendigkeit wird durch entweder synthetisch erzeugte oder elektronisch bearbeitete Instrumental- und Vokalklänge verstärkt. Der auf diese Weise erzielte Gesamtklang ist das Ergebnis einer elaborierten Soundtüftelei jenseits von vorgegebenen Konventionen oder gar Klischees - etwa im Hinblick auf Instrumentenbesetzungen, wie sie in mittelalterlicher Musik bevorzugt werden. Ein und derselbe Baustein erklingt zudem in den Wiederholungen immer wieder ein wenig anders. Die einzelnen Tonspuren wurden real eingespielt und nicht einfach geloopt. Was beim ersten Anhören noch als reduktionistisch, schlicht oder gar monoton wirken mag, erweist sich somit beim genauen Hinhören als keinesfalls weniger variantenreich als z. B. klangliche und rhythmische Strukturierungen in Minimal Music.

Die Stücke weisen zudem eine starke melodische Qualität auf. Die Aneinanderreihung von teils identischen, teils ähnlichen Motiven verdichtet sich zu Melodien in generell molltonalem Kontext. Rezeptionspsychologisch gesagt: Die steten, oft durch geringe Soundveränderungen variierten Motivwiederholungen führen zu einer Konditionierung des Gehörs. Die Motive gewinnen dadurch jene unverwechselbare Prägnanz und Eindringlichkeit, wie sie generell auch für gute Melodien im Refrainteil von Poptiteln kennzeichnend ist.

Die konkrete Ausformung der Musikstücke genügt den Kriterien der Kleinen Münze. Daran ändern auch die deutlich wahrnehmbaren Reminiszenzen an mittelalterliche Musik nichts. Die Anlehnungen an das eine oder andere Musikmodell des Mittelalters (Verwendung von Kirchentonarten, Bordunklänge, Quintparallelen) fungieren modellsemantisch als klingende Projektionsflächen für Traumbilder und Imaginationen von Mittelalter aus heutiger Sicht (Kreutziger-Herr 2003: 206f.). Musikalische Mittelalter-Topoi werden von Arcana in neue, der Dark Wave-Szene angemessene popmusikalische Zusammenhänge gebracht. Diese Art der Aneignung und kreativen Umsetzung von mittelalterlicher Klangsymbolik geht fraglos über das rein Handwerkliche und mithin gemeinfreie musikalische Vokabular hinaus. 
(2) Auf welche Weise nun Bushido Ausschnitte aus der Musik von Arcana verwendet, sei im Folgenden exemplarisch an dem Titel »Ghettorap hin, Ghettorap her « dargestellt. Bushido greift hier auf das Stück »Winds Of The Lost Soul«zurück. Der Ausschnitt entstammt dem Anfang des 4:30 Minuten langen Titels und hat eine Dauer von 20 Sekunden (Diagramm 1). Der Ausschnitt wird in stark beschleunigter Form verwendet. Dadurch verkürzt sich die Spieldauer auf 10,6 Sekunden. Das entspricht einem Verhältnis von 15:8 bzw. dem Intervall einer großen Septime aufwärts. Das Sample erklingt durchgängig vom Anfang bis zum Ende des Stücks als Tonschleife, im FadeOut jedoch verkürzt auf 7,4 Sekunden.

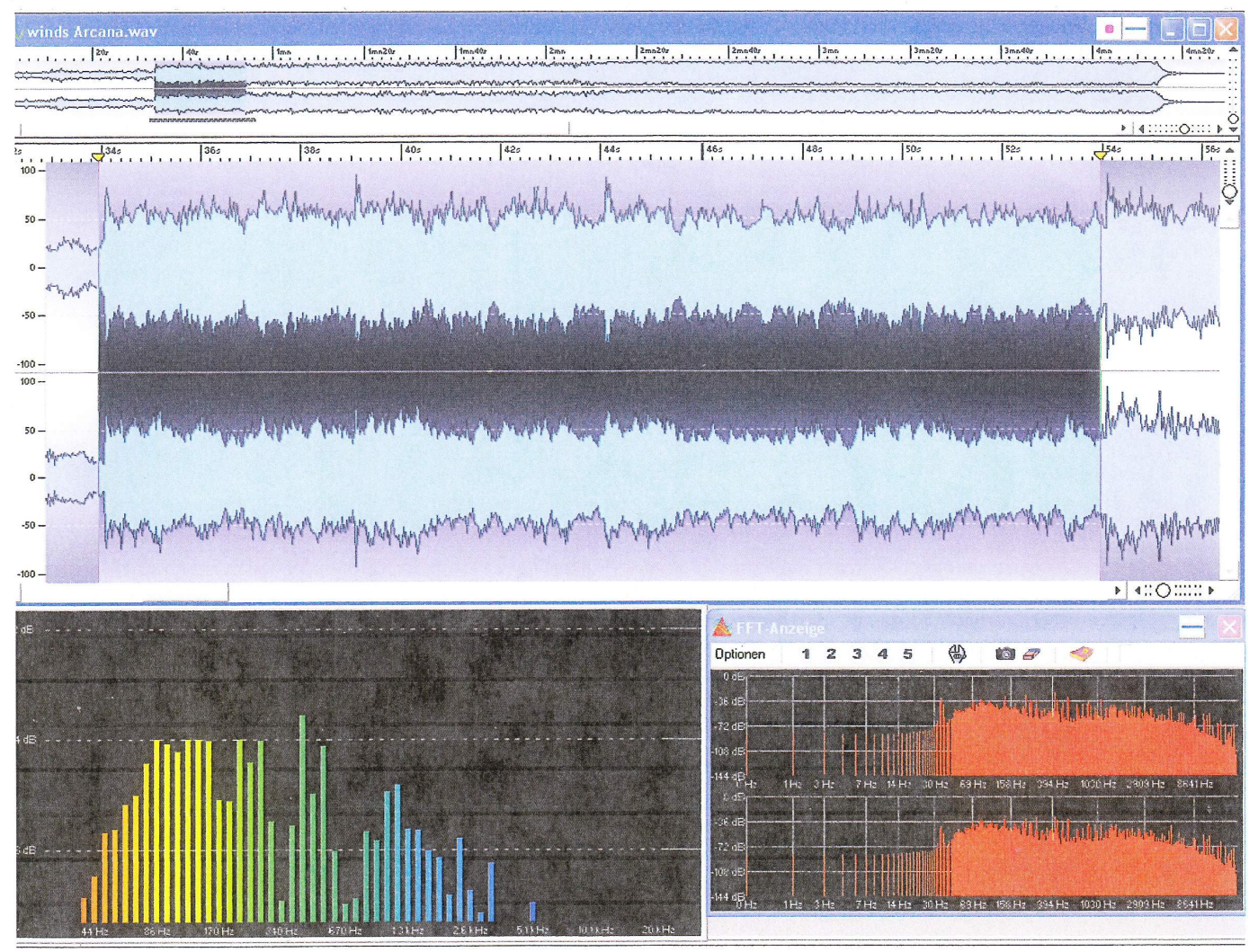

Diagramm 1: Arcana - »Winds Of The Lost Soul«

Der Ausschnitt beginnt mit einem markanten Röhrenglocken-Schlag zu liegenden Basstönen (Bordun Cis-Gis) und rhythmisch strukturierenden Figuren des Schlagzeugs. Die bereits zuvor gegenwärtige Vokalspur wird von einer neu einsetzenden Bläser-Linie mit Oboen-nahem Klang mehr in den Hintergrund des musikalischen Geschehens verschoben. Zu Beginn von Takt 4 wechselt der Bordunklang nach $E-A$ entsprechend den Tönen in der Oberstimme, bevor zum Taktende über den Vorhalt $H$ wieder Gis als Anfang des Loops erreicht wird. 
Die Oberstimme ist ebenso wie die dazu größtenteils in parallelen Quinten geführte 2. Stimme deutlich gegliedert. Sie besteht aus vier eintaktigen Teilen. Sie ergeben mit ihren unterschiedlichen, auf- und absteigenden Intervallen eine in sich geschlossene Tonfolge. Nicht zuletzt auch auf Grund der ständigen Wiederholung des Samples in »Ghettorap hin, Ghettorap her « erhalten sie den Status einer Melodie.

(3) Eine vergleichend-akustische Analyse mit dem Programm WaveLab $4.01 \mathrm{~b}$ bestätigt, dass die Übernahme als Sample $1: 1$, d.h. ohne eigenständige musikrelevante Bearbeitung erfolgte. Verlangsamt man das Bushido-Sample auf die Verlaufsgeschwindigkeit des Originals, dann sind die Hüllkurven der Amplitudenverläufe des Ausschnitts aus »Winds Of The Lost Soul« und dem Sample zu "Ghettorap hin, Ghettorap her « bis in die kleinsten Ausschläge hinein identisch (Diagramm 2). Einzig ein im Bushido-Beispiel nach 18,4 Sekunden zusätzlich getätigter Bassdrum-Schlag führt zu einem deutlichen Anstieg der Kurve. Er fehlt im Arcana-Beispiel.

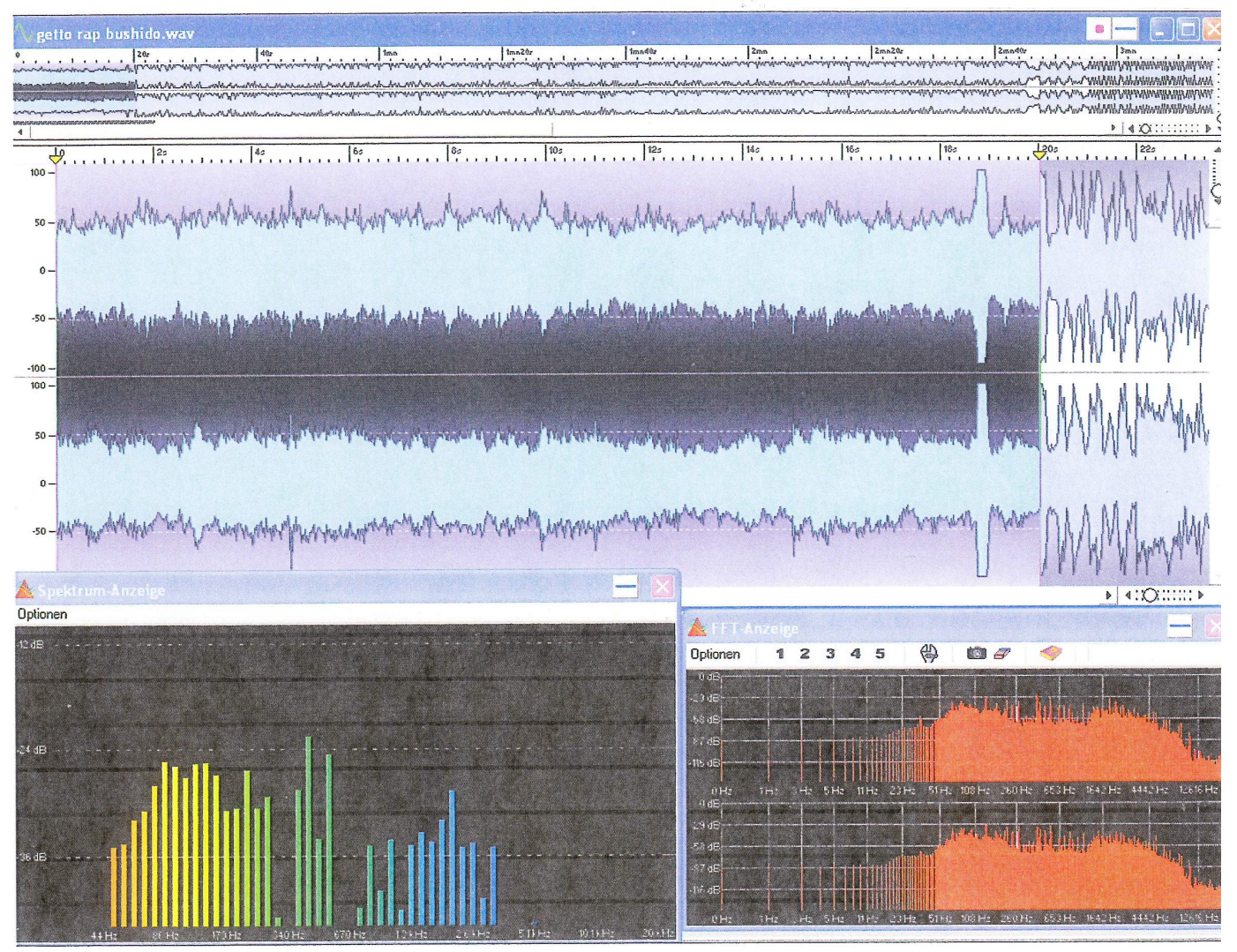

Diagramm 2: Bushido - »Ghettorap hin, Ghettorap her«

Auch die Anzeige der Spektren beider Ausschnitte bestätigt die Identität von Original und Sample. Die Frequenzbalken weisen große Übereinstimmung insbesondere in den musikalisch relevanten Frequenzbereichen auf. Bei 
Bushido wurde das gesamte Klangbild lediglich etwas aufgehellt. Das führt zu einer Reduktion der Balkenlänge in tiefster Lage (rot), zu einer leichten Ausdünnung der Balken in mittlerer Lage (grün) und zur Anhebung im Helligkeitsbereich (blau). Dieses Analyseergebnis wird durch die FFT-Anzeigen zusätzlich bestätigt.

Die mit Hilfe des Equalizers durchgeführte Modifizierung des Klangbildes bei Bushido hat einen einfachen Grund. Durch sie erfolgt eine Anpassung des Samples an eine der Rapmusik gemäße Nutzungs- und Wiedergabesituation. Arcanas Musik ist für die Beschallung größerer Räume in HiFi-Qualität konzipiert, Bushidos Stücke dagegen sollen ihre optimale Wirkung beim Anhören über kleine Wiedergabegeräte wie Autoradio, iPod u. ä. entfalten.

(4) Entscheidend ist nun, welche Rolle bzw. welche musikalische Funktion das Sample in Bushidos Stück übernimmt. Gibt der gesamplete Ausschnitt kaum mehr als einen klangtapetenartigen Hintergrund ab oder kommt ihm eine tragende Funktion für den Rapgesang Bushidos zu? Zunächst einmal ist festzuhalten, dass die Stücke von Bushido zwei Darbietungsbereiche enthalten, zum einen den von verschiedenen Schlagzeug-Figuren rhythmisch unterlegten Rapgesang und zum anderen das musikalische Geschehen. Im HipHop wird seit den Anfängen bei Grandmaster Flash gesamplet. Die Samples bestehen allerdings in der Regel aus Fragmenten mit zwei bis drei Sekunden Dauer, und sie werden durch raffinierte Schichtungen im Mehrspurverfahren zu einer neuen Musik geformt. Diese kann allenfalls von Kennern auf ihre originalen Bestandteile hin erfasst werden.

Im Vergleich dazu folgt der Einsatz des Samples bei Bushido einem sehr einfachen Verarbeitungsmuster. Darum ist das Sample hier ohne weiteres im Hinblick auf das zugrunde liegende Original zu identifizieren. Selbst die Transposition durch Beschleunigung der Abspielgeschwindigkeit lässt die musikalische Grundsubstanz unverändert durchscheinen. Die gestalterische Charakteristik des Ausschnitts erweist sich als hochgradig transpositionsresistent. Genau das aber ist ein entscheidender Faktor für jedwede »gute Gestalt« im Sinn der Wahrnehmungspsychologie.

Die Identifikation des Samples wird zudem dadurch erleichtert, dass es auch solistisch erklingt. Und das nicht nur in der Introduktion, bevor Sprechgesang zu Drumset-Schlägen einsetzt, sondern auch am Schluss. Bis zum letzten Fade-Out ist es hier dreimal zu hören. Bereits dieser Umstand spricht gegen eine Verwendung des Samples lediglich als Klangtapete. Hinzu kommt, dass der Rap-Vortrag von Bushido überhaupt erst durch den geloopten Arcana-Ausschnitt eine musikalische Darbietungsebene erhält. Das wird 
auch im CD-Booklet so gesehen. Allerdings ist hier fälschlicher Weise Bushido als Urheber für die Musik genannt.

Die kontinuierlichen Sample-Wiederholungen innerhalb des Stücks stärken seinen Wiedererkennungswert. Das gilt selbst dann, wenn die Aufmerksamkeit beim Anhören auf die Rap-Texte gerichtet ist. Für diejenigen, die dem Inhalt der Texte - in denen mit deutlicher Wiederholungsredundanz gearbeitet wird - nicht immer konzentriert folgen wollen, gewinnt die musikalische Darbietungsebene eine zumindest gleichrangige Bedeutung. Ihre Beziehung zur Textebene ist durch den Kontrast bestimmt. Hier handelt es sich um eine unverkennbare, klanglich markante und weite Assoziationsräume eröffnende Musik gegenüber der durch Agilität, Härte und verbale Provokation gekennzeichneten Rap-Deklamation. Gerade dieser Kontrast ist es, der die Eindringlichkeit des Rapgesangs erheblich steigert. Würde die von Arcana komponierte Dark Ambient-Musik gegenüber den Rapdarbietungen von Bushido verblassen, dann wäre das ein großer Verlust: Dem Stück von Bushido fehlte dann eine musikalische Dimension.

Alles in allem macht die Analyse deutlich: Der von der Klägerseite erhobene Plagiatsvorwurf ist nicht von der Hand zu weisen. Denn das Sample, das Bushido seinem Rapgesang als musikalisches Geschehen zugrunde gelegt hat, ist urheberrechtlich geschützt. Fremdes geistiges Eigentum wurde nutzbringend verwendet, ohne den Urheber, die Gruppe Arcana, um Erlaubnis zu fragen und am Gewinn zu beteiligen.

\section{Schlussbemerkung}

Forensische Popmusikanalyse bildet die Grundlage dafür, Musik gemäß den Richtlinien der UNESCO als immaterielles geistiges Eigentum zu schützen. Der Annahme, heutzutage sei frei verfügbar, was z.B. via Internet und MP3Files abrufbar ist, kann damit ein Riegel vorgeschoben werden. Allerdings gilt es einige Grundsätze zu beherzigen, damit die Analyse zu angemessenen Ergebnissen führt. Popmusik kann und darf nicht nach Kriterien der westlichen Kunstmusik betrachtet und bewertet werden. Kompositorisch gesehen besteht ihre Aufgabe darin, durch eine bewusste Reduktion der musikstrukturellen Mittel möglichst eingängige und zugleich prägnante Gestalten zu schaffen. Gekonnter Minimalismus wird zum Qualitätskriterium und Erfolgsrezept. Als Anschauungsmodell dafür mag etwa das Lied »Da Da Da« von Trio ebenso gelten wie so manche Titel von Modern Talking. Das vermeintlich Triviale entfaltet schon dann seine individuelle Wirkung, wenn es in Kleinigkeiten von dem abweicht, was in den musikalischen Lehrbüchern 
notiert und im allgemein verfügbaren Liedgut gegenwärtig ist. Jedes Intervall, jede binnenrhythmische Veränderung, jede neue Soundvariante ist hier von Belang, aber ebenso auch jede Wiederholung eines rhythmisch, klanglich, harmonisch und melodisch gestalteten Motivs. Das eine wie das andere setzt Entscheidungen voraus. Und genau darin besteht die Arbeit des Komponierens. Mag man auch darüber streiten, ob ein einziger elektronischer Sound von Kraftwerk bereits Urheberrechtsschutz genießen sollte, ${ }^{5}$ so bleibt doch unbestritten, dass die Popmusikproduktionen der letzten Jahre nicht allesamt gemeinfreies Kulturgut sein können.

\section{Literatur}

Brösche, Thomas (1998). »Urheberrecht.«In: Die Musik in Geschichte und Gegenwart. Sachteil Bd. 9. Hg. v. Ludwig Finscher. Kassel u.a. / Stuttgart u. Weimar: Bärenreiter / Metzler (2. Aufl.), Sp. 1203-1213.

Elster, Alexander (1921). Gewerblicher Rechtsschutz. Berlin: De Gruyter.

Kreutziger-Herr, Annette (2003). Ein Traum von Mittelalter. Die Wiederentdeckung mittelalterlicher Musik in der Neuzeit. Köln: Böhlau.

Pendzich, Marc (2004). Von der Coverversion zum Hit-Recycling. Historische, ökonomische und rechtliche Aspekte eines zentralen Phänomens der Pop- und Rockmusik. Münster: Lit.

Rösing, Helmut (2002). »>Popularmusikforschung in Deutschland - von den Anfängen bis zu den 1990er Jahren. «In: Musikwissenschaft und populäre Musik: Versuch einer Bestandsaufnahme. Hg. v. Helmut Rösing, Albrecht Schneider und Martin Pfleiderer (= Hamburger Jahrbuch für Musikwissenschaft 19). Frankfurt/M. u. a.: Lang, S. 13-35.

Schneider, Albrecht (2002). »Klanganalyse als Methodik der Popularmusikforschung. «In: Musikwissenschaft und populäre Musik: Versuch einer Bestandsaufnahme. Hg. v. Helmut Rösing, Albrecht Schneider und Martin Pfleiderer (= Hamburger Jahrbuch für Musikwissenschaft 19). Frankfurt/M. u. a.: Lang, S. 107-129.

Stoffer, Thomas H. (1996). »Mentale Repräsentation musikalischer Strukturen. « In: Zeitschrift für Semiotik 18, S. 213-234.

Wicke, Peter (Hg.) (2001). Rock und Popmusik (= Handbuch der Musik im 20. Jahrhundert 8). Laaber: Laaber.

Wicke, Peter (2002). »Popmusik in der Theorie. Aspekte einer problematischen Beziehung. «In: Musikwissenschaft und populäre Musik: Versuch einer Bestandsaufnahme. Hg. v. Helmut Rösing, Albrecht Schneider und Martin Pfleiderer (= Hamburger Jahrbuch für Musikwissenschaft 19). Frankfurt/M. u.a.: Lang, S. 61-73.

Wiora, Walter (1961). Die vier Weltalter der Musik. Ein universalhistorischer Entwurf. Stuttgart: Kohlhammer.

5 Vgl. dazu die Verlautbarung der Pressestelle des BGH Karlsruhe vom 20. Dezember 2008: »Metall auf Metall« (Az. I ZR 112/06). 


\title{
Diskographie
}

Arcana (2000). The Last Embrace. Cold Meat Industry (Schweden) CMI 79. Arcana (2002). Inner Pale Sun. Cold Meat Industry (Schweden) CMI 121. Arcana (2004). The New Light. Erebus Odora (Schweden) 005.

Bega, Lou (1999). Mambo No. 5 - A Little Bit Of... BMG EAN 74321658012.

Bushido (2005). Carlo Cokxxx Nutten II. Urban/ersguterjunge 987 080-1.

Die Doofen (1994). Lieder, die die Welt nicht braucht. BMG-Ariola 7432127206-2.

Hall, Kristen (1994). Be Careful What You Wish For. High Street Records 10326.

Lippe, Jürgen von der (1990). Humor ist Humor. BMG-Ariola 261126.

Marshall, Amanda (1995). »Let It Rain«. Auf CD V.A. - Kuschelrock 10. Sony SMM 4840002.

Prado, Perez (1990). Perez >Prez Prado: King Of Mambo. RCA EAN 003562904 2421.

\begin{abstract}
Forensic analysis of pop music is usually comparative. Main topic is the question about the individual creative parts of one composition, using material of a formerly produced and copyrighted piece of music. To prove the violation of author's copyright it seems unavoidable to point out that (1) the original composition has creative qualities and that (2) the one or other of these creative elements is an essential part of the newer music. The analysis of four examples of presumptive plagiarism makes it obvious that in pop music the criterion for creativity is due to minimal changes in working up nearly identical musical elements.
\end{abstract}

